\title{
UNSTEADY ASYMPTOTIC SOLUTIONS OF THE TWO-DIMENSIONAL EULER EQUATIONS
}

\author{
$\mathrm{BY}$ \\ RADHAKRISHNAN SRINIVASAN \\ Director's Unit, National Aerospace Laboratories, Bangalore-560017, India
}

\begin{abstract}
A technique is described for deducing a class of unsteady asymptotic solutions of the two-dimensional Euler equations. In contrast to previously known analytical results, the vorticity function $[\omega(x, y, t)]$ for these solutions has a complicated dependence on the spatial coordinates $(x, y)$ and time $(t)$. The results obtained are in implicit form and are valid in those regions of space and time where $t \omega \rightarrow 0^{+}$or $t \omega \rightarrow+\infty$. These asymptotic solutions may be split into an unsteady, two-dimensional and irrotational basic flow and a disturbance that is strongly nonlinear at appropriate locations within the domain of validity. The generality and complexity of these solutions make them theoretically interesting and possibly useful in applications.
\end{abstract}

1. Introduction and formulation. Let $\psi(x, y, t)$ denote the stream function for the two-dimensional flow of an inviscid incompressible fluid, where $(x, y)$ are Cartesian coordinates and $t$ is the time. The velocity components are given by $(u, v)=\left(\psi_{y},-\psi_{x}\right)$ (the subscripts denote partial derivatives). This flow is governed by the Euler equations as given below:

$$
\omega_{t}+\psi_{y} \omega_{x}-\psi_{x} \omega_{y}=0
$$

where $\omega(x, y, t)$ is the vorticity, defined by

$$
\omega=-\left(\psi_{x x}+\psi_{y y}\right)
$$

Unsteady two-dimensional analytical solutions of the above nonlinear system, whether exact or asymptotic, are very difficult to obtain; see, for example, Batchelor [1] for some of the known results on the Euler equations. Many of the known unsteady exact solutions of $(1 \mathrm{a}, \mathrm{b})$ are listed in the review articles of Berker [2] and, more recently, Wang [3]; a common feature of these solutions which makes them limited in applications is that the vorticity function $\omega$ has a rather simple dependence on $(x, y, t)$. Apart from the case of constant or zero vorticity, some examples of the known unsteady exact solutions of (1a, b) listed in $[2,3]$ are for the cases (a) $\omega=f\left(x^{2}+y^{2}, t\right)$, (b) $\omega=f(y / x, t)$, (c) $\omega=f(\xi)$, where $\xi \equiv x+g(t) y$, (d) $\omega=f(x, y) / t$, and (e) $\omega=f(x, t)$. The function $f$ may be

Received June 28, 1993.

1991 Mathematics Subject Classification. Primary 76.

(c)1996 Brown University 
determined upon substitution into (1a, b), as shown in Appendix A. Recently, Ranger $[4,5]$ has obtained complex solutions of the steady-state Navier-Stokes equations in implicit form.

The purpose of this paper is to determine, in implicit form, a class of unsteady asymptotic solutions of $(1 \mathrm{a}, \mathrm{b})$ for which the vorticity function $\omega$ has a complicated dependence on $(x, y, t)$. Apart from the theoretical insight that these solutions provide, their generality and complexity may make them interesting in applications as well.

We now describe our technique. The idea is to employ the hodograph transformation, as follows. Let the vorticity $(\omega)$ and $x$ be independent and dependent variables respectively, and define

$$
\psi(x, y, t)=\Psi(\omega, y, t) \quad \text { and } \quad x=X(\omega, y, t) .
$$

From (2), it follows that

$$
\omega_{x}=\left(1 / X_{\omega}\right), \quad \omega_{t}=-\left(X_{t} / X_{\omega}\right), \quad \omega_{y}=-\left(X_{y} / X_{\omega}\right)
$$

and

$$
\omega_{x x}=-\left(X_{\omega \omega} / X_{\omega}^{3}\right), \quad \omega_{y y}=X_{y}^{2} \omega_{x, r}+\left(2 X_{y} X_{\omega y} / X_{\omega}^{2}\right)-\left(X_{y y} / X_{\omega}\right) .
$$

Note that the transformation (2) is legal provided $X_{\omega} \neq 0$ anywhere, as is apparent from $(3 \mathrm{a}, \mathrm{b})$; it is therefore assumed that $\omega$ is a smooth function of the coordinates in the (as yet undefined) flow domain. Obviously, $\omega$ must depend on $x$; it cannot, for example, be a constant. Our strategy is to proceed formally with the asymptotic solution of (1a, b) for $\Psi$ and $X$, and then restrict the flow domain to such regions of space and time that (2) is legal.

Upon inserting (2) into (1a) and using (3a), we obtain $\Psi_{y}=-\left(\omega_{t} / \omega_{x}\right)=X_{l}$. Hence

$$
\Psi(\omega, y, t)=\int_{0}^{y} X_{t}(\omega, s, t) d s-A(\omega, t)
$$

where $A(\omega, t)$ is an arbitrary function. Equation (1b) may be written as

$$
\Psi_{\omega \omega}\left(\omega_{x}^{2}+\omega_{y}^{2}\right)+\Psi_{\omega}\left(\omega_{x x}+\omega_{y y}\right)+\Psi_{y y}+2 \Psi_{\omega y} \omega_{y}=-\omega
$$

where we substitute for the derivatives of $\omega$ from $(3 a, b)$ and for the derivatives of $\Psi$ from appropriate differentiation of (4).

Equation (5) may be interpreted as a nonlinear integro-differential equation for $X(\omega, y, t)$, given an arbitrary $A(\omega, t)$; however, in general, this approach turns out to be analytically intractable. A more useful approach is to guess a suitable function $X(\omega, y, t)$ such that (5) can be solved for $A(\omega, t)$, either exactly or asymptotically in some flow domain. Some well-known unsteady exact solutions of (5) that can be obtained in this manner are for (a suitably inverted form of) the cases (a)--(e) described earlier.

If our objective is restricted to determining exact solutions only, then Eq. (5) does not seem to be useful in generating new results. However, it will be shown in the ensuing section that new unsteady asymptotic solutions of the Euler equations can be obtained in closed form from (5); the vorticity for these solutions is a more complex function than those described in (a)-(e) above. It should be emphasized that, in general, these are not necessarily "weakly nonlinear" solutions (which are obtained by solving the linearized equations for small perturbations of known exact solutions). 


\section{Asymptotic solutions.}

\subsection{Solution procedure. We let}

$$
X(\omega, y, t)=-y h(\omega, t)-g(\omega, t)
$$

where $h$ and $g$ are functions to be determined. Upon substituting (6) into (4) and then (4) and $(3 \mathrm{a}, \mathrm{b})$ into the left-hand side of (5) and multiplying throughout by $\left(y h_{\omega}+g_{\omega}\right)^{3}$, we obtain a cubic polynomial in $y$. The coefficients of each power of $y$ must vanish, and hence we obtain the following four equations for the three unknown functions $A$, $h$, and $g$ :

Coefficient of $y^{(0}$ :

$$
A_{\omega \omega} g_{\omega}\left(1+h^{2}\right)-A_{\omega}\left[g_{\omega \omega}\left(1+h^{2}\right)-2 h h_{\omega} g_{\omega}\right]-2 h g_{\omega}^{2} g_{\omega t}+\left(h_{t}-\omega\right) g_{\omega}^{3}=0
$$

Coefficient of $y$ :

$$
\begin{aligned}
A_{\omega \omega} h_{\omega}\left(1+h^{2}\right)-A_{\omega}\left[h_{\omega \omega}(1\right. & \left.\left.+h^{2}\right)-2 h h_{\omega}^{2}\right]+\left(1+h^{2}\right)\left(g_{\omega} g_{\omega \omega t}-g_{\omega \omega} g_{\omega t}\right) \\
& -2 h g_{\omega}^{2} h_{\omega t}-2 h h_{\omega} g_{\omega} g_{\omega t}+3 h_{\omega} g_{\omega}^{2}\left(h_{t}-\omega\right)=0,
\end{aligned}
$$

Coefficient of $y^{2}$ :

$$
\begin{aligned}
\left(1+h^{2}\right)\left[h_{\omega \omega t} g_{\omega}+2 g_{\omega \omega t} h_{\omega}\right. & \left.-h_{\omega t} g_{\omega \omega}-2 g_{\omega t} h_{\omega \omega}\right] \\
& -6 h h_{\omega t} h_{\omega} g_{\omega}+6 h_{\omega}^{2} g_{\omega}\left(h_{t}-\omega\right)=0,
\end{aligned}
$$

Coefficient of $y^{3}$ :

$$
\left(1+h^{2}\right)\left[h_{\omega \omega t} h_{\omega}-h_{\omega t} h_{\omega \omega}\right]-2 h h_{\omega}^{2} h_{\omega t}+2\left(h_{t}-\omega\right) h_{\omega}^{3}=0 .
$$

In general, one expects that a solution to the over-determined system of Eqs. (7)-(10) does not exist. However, this is not necessarily true; for example, given a solution $h(\omega, t)$ of (10), it is easy to verify that $g=h$ is a solution of (9) [which merely reduces to a multiple of (10)]. Upon setting $g=h$ in Eqs. (7) and (8) \{and using (10) in (8)\}, we find that these reduce to identical first-order ordinary differential equations for $A_{\omega}$, the solution of which is straightforward. But this solution, although not previously known explicitly in this form, is perhaps not very interesting, being equivalent to the previously known result (b) of Sec. 1 (as can be seen upon setting $g=h$ in (6)).

In this paper, we shall be concerned with determining a class of asymptotic solutions of $(7)-(10)$, valid as $h(\omega, t) \rightarrow 0^{+}$. Here the emphasis is on the asymptotic validity of Eq. (6) and hence (7)-(10), in the above limit. We shall not be concerned here with the possibility of obtaining new exact solutions of (7)-(10).

Make the transformation

$$
h(\omega, t)=H(\lambda) \quad \text { where } \lambda=\omega t .
$$

Equation (10) then reduces to the following nonlinear ordinary differential equation:

$$
\begin{aligned}
\left(1+H^{2}\right)\left[\lambda H^{\prime} H^{\prime \prime \prime}-\lambda\left(H^{\prime \prime}\right)^{2}+H^{\prime} H^{\prime \prime}\right]-2 H\left(H^{\prime}\right)^{2}\left(H^{\prime}+\lambda H^{\prime \prime}\right) & \\
& +2 \lambda\left(H^{\prime}-1\right)\left(H^{\prime}\right)^{3}=0
\end{aligned}
$$


where the primes imply differentiation. We seek asymptotic solutions of (12) as $H \rightarrow 0^{+}$ in the form

$$
H=H_{0}(\lambda)+H_{1}(\lambda)+E(\lambda) \quad \text { where } 0<H_{1} \ll H_{0} \ll 1 \quad \text { and } \quad 0<H_{1}^{\prime} \ll H_{0}^{\prime} \ll 1 \text {, }
$$

and $E(\lambda)$ is an error term satisfying $E=o\left(H_{1}\right)$. We may verify that $H_{0}$ and $H_{1}$ satisfy

$$
\lambda\left[H_{0}^{\prime} H_{0}^{\prime \prime \prime}-\left(H_{0}^{\prime \prime}\right)^{2}\right]+H_{0}^{\prime} H_{0}^{\prime \prime}=0
$$

and

$$
\lambda\left[H_{0}^{\prime} H_{1}^{\prime \prime \prime}+H_{0}^{\prime \prime \prime} H_{1}^{\prime}-2 H_{0}^{\prime \prime} H_{1}^{\prime \prime}\right]+H_{0}^{\prime} H_{1}^{\prime \prime}+H_{0}^{\prime \prime} H_{1}^{\prime}=2 \lambda\left(H_{0}^{\prime}\right)^{3} .
$$

One set of solutions of $(14 \mathrm{a}, \mathrm{b})$ is given by

$$
H_{0}(\lambda)=\alpha(k+1)^{-1} \lambda^{k+1}
$$

and

$$
H_{1}(\lambda)=2 \alpha^{2}(k+2)^{-2}(2 k+3)^{-1} \lambda^{2 k+3},
$$

where $\alpha$ and $k$ are any constants satisfying $\alpha>0$ and $k \notin[-2,0]$. Without loss of generality, we may take $\lambda>0$; for example, we may consider the odd extensions of the functions $H(\lambda), G_{n}(\lambda)$, and $A(\omega, t)$ to negative $\lambda$. From $(15 \mathrm{a}, \mathrm{b})$, we conclude that the expansion (13) is a formally valid asymptotic solution of (12) provided

$$
\lambda \rightarrow 0^{+} \quad \text { and } \quad k>0 \quad \text { or } \quad \lambda \rightarrow+\infty \text { and } k<-2 .
$$

We shall develop our asymptotic theory based on either of the requirements in $(16 a, b)$. In Appendix B, it is shown formally that the error term in (13) satisfies

$$
E(\lambda)=O\left(\lambda^{3 k+5}\right) \text { if }(16 \mathrm{a}) \text { or }(16 \mathrm{~b}) \text { holds. }
$$

For any $H(\lambda)$ satisfying (12), we seek solutions of (9) in the form

$$
g(\omega, t)=G_{n}(\lambda) t^{n} \equiv g_{n}(H) t^{n}
$$

where the argument of $g_{n}$ is $H=H(\lambda), n$ is an arbitrary real constant and the time $t$ is assumed positive henceforth. The problem for $g_{n}(H)$ turns out to be simpler; upon substitution into (9), the coefficient of $g_{n}^{\prime}(H)$ cancels out because of (12), and we obtain

$$
g_{n}^{\prime \prime \prime}+\left[\frac{3 H^{\prime \prime}}{2\left(H^{\prime}\right)^{2}}+\frac{2 n+3}{2 \lambda H^{\prime}}\right] g_{n}^{\prime \prime}=0 \text {. }
$$

Note that the primes refer to differentiation with respect to the arguments of the functions $g_{n}$ and $H$. One solution of the above equation is clearly $g_{n}=H$; we ignore this solution and take

$$
G_{n}^{\prime}(\lambda)=H^{\prime}(\lambda) g_{n}^{\prime}(H)=c H^{\prime}(\lambda) \int^{\lambda}\left(H^{\prime}\right)^{-1 / 2} \lambda^{-n-(3 / 2)} d \lambda
$$


where $c$ is an arbitrary, nonzero constant. Equation (7) may be easily solved for $A_{\omega}$, and we obtain

$$
A_{\omega}=\left[\frac{t G_{n}^{\prime}}{1+H^{2}}\right]\left[f_{n}(t)+t^{2 n-1} \int^{\lambda}\left\{2 \lambda H G_{n}^{\prime \prime}+2(n+1) H G_{n}^{\prime}-\lambda\left(H^{\prime}-1\right) G_{n}^{\prime}\right\} d \lambda\right]
$$

where $f_{n}(t)$ is an arbitrary function.

We have solved (10), (9), and (7) for the three unknown functions $h, g$, and $A$. We now examine the conditions under which Eq. (8) is satisfied by these results, at least asymptotically to a few leading orders as $H(\lambda) \rightarrow 0^{+}$. Henceforth, use of the asymptotic equality $\operatorname{sign}(\sim)$ or the ordering symbols $(\gg, \ll, o$, and $O$ ) implies that either one of Eqs. (16a, b) holds. Using (13) and (15a, b) in (19) yields the following asymptotic expansions for $G_{n}^{\prime}$ :

$$
\begin{gathered}
G_{n}^{\prime}(\lambda) \sim-\frac{2 c \alpha^{1 / 2} \lambda^{-n+(k-1) / 2}}{k+1+2 n}-\frac{2 c \alpha^{3 / 2}(3 k-2 n+7) \lambda^{-n+(3 k+3) / 2}}{(k+2)^{2}(k+3-2 n)(k+1+2 n)} \\
\text { for }(k+1+2 n) \neq 0 \text { and }(k+3-2 n) \neq 0, \\
G_{n}^{\prime}(\lambda) \sim c \alpha^{1 / 2} \lambda^{k} \log (\lambda)\left[1+2 \alpha(k+2)^{-2} \lambda^{k+2}\right] \quad \text { for } k+1+2 n=0,
\end{gathered}
$$

and

$$
\begin{array}{r}
G_{n}^{\prime}(\lambda) \sim-c \alpha^{1 / 2}\left[(k+2)^{-1} \lambda^{-2}+\alpha(k+2)^{-2} \lambda^{k} \log (\lambda)+2 \alpha(k+2)^{-3} \lambda^{k}\right] \\
\text { for } k+3-2 n=0 .
\end{array}
$$

In $(21 \mathrm{a}, \mathrm{b}, \mathrm{c})$ we have only included the required number of terms for the purposes of our analysis; the error terms may be estimated from (17). Using (13), (15), and (21) in (20) yields

$$
\begin{gathered}
A_{\omega}(\omega, t) \sim t f_{n}(t) G_{n}^{\prime}(\lambda)+\frac{8 \alpha c^{2} t^{2 n} \lambda^{k+1-2 n}}{(k+1+2 n)^{2}(k+3-2 n)} \\
\quad \text { for }(k+1+2 n),(k+3-2 n) \neq 0, \\
A_{\omega}(\omega, t) \sim t f_{n}(t) G_{n}^{\prime}(\lambda)+\alpha c^{2}(k+2)^{-1} t^{2 n} \lambda^{2 k+2}\{\log (\lambda)\}^{2} \quad \text { for } k+1+2 n=0,
\end{gathered}
$$

and

$$
A_{\omega}(\omega, t) \sim \alpha c^{2}(k+2)^{-2} t^{2 n} \lambda^{-2} \log (\lambda)+t f_{n}(t) G_{n}^{\prime}(\lambda) \quad \text { for } k+3-2 n=0 .
$$

In Eqs. $(22 \mathrm{a}, \mathrm{c})$ we retain, for our purposes, only the leading term of the expansions for $G_{n}^{\prime}(\lambda)$ given in $(21 \mathrm{a}, \mathrm{c})$; in $(22 \mathrm{~b})$, we retain the leading two terms of $G_{n}^{\prime}(\lambda)$ as given in (21b).

Denote the left-hand side of $(8)$ as $R_{8}(\lambda, t)$; we clearly need the coefficients in the asymptotic expansion of this residual to vanish, in order to formally validate our results. Upon substituting the expansions obtained above for $h, g_{\omega}$, and $A_{\omega}$ into the left-hand side of (8), we obtain the following results (the details are straightforward and omitted here). 
Case (i). $k+1+2 n \neq 0, k+3-2 n \neq 0$.

The residual from (8) reduces to

$$
\begin{gathered}
R_{8}(\lambda, t) \sim c \alpha^{3 / 2} \lambda^{-n+(3 k-3) / 2} t^{3} f_{n}(t)+(0) \lambda^{k-2 n-2} t^{2 n+2} \\
+(0) \lambda^{2 k-2 n} t^{2 n+2}
\end{gathered}
$$

where the above notation indicates that terms having the factor $(0)$, namely those of $O\left(\lambda^{k-2 n-2} t^{2 n+2}\right)$ and $O\left(\lambda^{2 k-2 n} t^{2 n+2}\right)$, have cancelled themselves out. The cancelled terms of $O\left(\lambda^{k-2 n-2} t^{2 n+2}\right)$ arise from the term $\left(g_{\omega} g_{\omega \omega t}-g_{\omega \omega} g_{\omega t}\right)$ in (8).

Case (ii). $k+1+2 n=0$.

We obtain the following residual from (8):

$$
\begin{gathered}
R_{8}(\lambda, t) \sim(0) \lambda^{2 k-1}\left[t^{2 n+2}\{\log (\lambda)\}^{2}+\left\{t^{2 n+2}+t^{3} f_{n}(t)\right\} \log (\lambda)\right] \\
+c \alpha \lambda^{2 k-1}\left[\alpha^{1 / 2} t^{3} f_{n}(t)-c t^{2 n+2}\right] \\
+(0) \lambda^{3 k+1}(\log \lambda)^{2} t^{2 n+2}
\end{gathered}
$$

Case (iii). $k+3-2 n=0$.

The residual from (8) reduces to

$$
\begin{aligned}
& R_{8}(\lambda, t) \sim(0) t^{2 n+2}\left[\lambda^{-5}+\lambda^{k-3} \log (\lambda)\right] \\
& +c \alpha^{3 / 2} \lambda^{k-3}\left[t^{3} f_{n}(t)+2 c \alpha^{1 / 2}(k+2)^{-2} t^{2 n+2}\right] .
\end{aligned}
$$

From (23)-(25) we see that the leading term of $R_{8}(\lambda, t)$ does cancel out if, for example, we take $f_{n}(t) \equiv 0$ in all cases. Hence to leading order, the expansions we have obtained for $\Psi$ and $X$ are indeed formally valid. But this leading-order result is not interesting for reasons explained below. To ensure that our results are interesting, we need to consider a sufficient number of terms in the expansions for $\Psi$ and $X$ such that the $(-\omega)$ term on the right-hand side of (5) cancels out; otherwise, we have merely obtained an asymptotic solution of $\psi_{x x}+\psi_{y y}=0$ (note that the left-hand side of (5) is precisely $\psi_{x x}+\psi_{y y}$ ). Secondly, we need to consider the region $y h=O(g)$ on the right-hand side of $(6)$, so that the leading part of $\omega(x, y, t)$ does not reduce to one of the known solutions (b) or (e) of Sec. 1. Now, the $(-\omega)$ term in the right-hand side of (5) translates into the last terms of Eqs. (7)--(10) which explicitly involve $-\omega$ as a coefficient; that is, $-\omega g_{\omega}^{3}$ in (7), etc. In order to meet the above two requirements, we must consider a sufficient number of terms in our expansions such that the last terms in (7)-(10) get cancelled out to at least leading order.

It can easily be verified that it is sufficient for our purposes if $R_{8}(\lambda, t)$ vanishes to the orders indicated in (23)-(25); for example, if case (i) holds, the last term of (8) given by $\left\{-3 \omega h_{\omega} g_{\omega}^{2}\right\}$ is $O\left(\lambda^{2 k-2 n} t^{2 n+2}\right)$. It follows from these equations that we need

$$
\begin{aligned}
& f_{n}(t) \equiv 0 \quad \text { if }(23) \text { holds, } \\
& f_{n}(t)=c \alpha^{-1 / 2} t^{2 n-1} \quad \text { if (24) holds, }
\end{aligned}
$$

and

$$
f_{n}(t)=-2 c \alpha^{1 / 2}(k+2)^{-2} t^{2 n-1} \quad \text { if }(25) \text { holds. }
$$


Note from (4), (6), (11), and (18) that the stream function $\Psi$ is given by

$$
\Psi(\omega, y, t)=-\left[y^{2} \lambda H^{\prime}(\lambda) /(2 t)\right]-y t^{n-1}\left[\lambda G_{n}^{\prime}(\lambda)+n G_{n}(\lambda)\right]-A(\omega, t) .
$$

To obtain the expansions for $G_{n}(\lambda)$ and $A(\omega, t)$, we integrate Eqs. (21) and (22) term by term, omitting any constants of integration. We are now in a position to state our asymptotic results for $\Psi$ and $X$, which can be obtained upon inserting suitable expansions for $H(\lambda), G_{n}(\lambda)$, and $A(\omega, t)$ into the right-hand sides of (29) and (6).

2.2. Asymptotic results.

Case (i). Equations (23) and (26) hold.

We state our asymptotic results for the two subcases $k+1-2 n \neq 0$ and $k+1-2 n=0$. Case (ia). $k+1-2 n \neq 0$ and (23), (26) hold.

Define $y_{1}, \phi_{1}(\lambda), \phi_{2}(\lambda)$, and $F_{1}\left(\lambda, y_{1}\right)$ by

$$
\begin{gathered}
y_{1}=y t^{-n} \lambda^{n+(k+1) / 2}, \\
\phi_{1}(\lambda)= \begin{cases}\log \lambda & \text { if } 3 k+5-2 n=0, \\
2 /(3 k+5-2 n) & \text { if } 3 k+5-2 n \neq 0,\end{cases} \\
\phi_{2}(\lambda)= \begin{cases}\log \lambda & \text { if } k+2-2 n=0, \\
1 /(k+2-2 n) & \text { if } k+2-2 n \neq 0,\end{cases}
\end{gathered}
$$

and

$$
F_{1}\left(\lambda, y_{1}\right)=\lambda^{-2 n}\left[\frac{2 c \alpha^{1 / 2}(k+1) y_{1}}{(k+1+2 n)(k+1-2 n)}-\left(\alpha y_{1}^{2} / 2\right)\right]
$$

We obtain the following expansions for $\Psi$ and $X$ from (29) and (6); for convenience, we state our results in terms of the variable $y_{1}$ rather than $y$.

$$
\begin{aligned}
\Psi(\omega, y, t) & \sim t^{2 n-1} F_{1}\left(\lambda, y_{1}\right) \\
& -t^{2 n-1} \lambda^{k+2-2 n}\left[K_{1} \phi_{2}(\lambda)-K_{2}\left\{n \phi_{1}(\lambda)+1\right\} y_{1}+K_{3} y_{1}^{2}\right],
\end{aligned}
$$

where the constants $K_{i}$ are defined by

$$
\begin{aligned}
& K_{1}=8 \alpha c^{2} /\left[(k+1+2 n)^{2}(k+3-2 n)\right], \\
& K_{2}=2 c \alpha^{3 / 2}(3 k-2 n+7) /\left[(k+2)^{2}(k+3-2 n)(k+1+2 n)\right],
\end{aligned}
$$

and

$$
K_{3}=\alpha^{2} /(k+2)^{2}
$$

In (30a), $\lambda(x, y, t)\{=t \omega(x, y, t)\}$ is defined by the implicit relationship

$$
\begin{gathered}
x=X(\omega, y, t) \sim-t^{n} \lambda^{-n+(k+1) / 2}\left[\frac{\alpha y_{1}}{k+1}-\frac{4 c \alpha^{1 / 2}}{(k+1+2 n)(k+1-2 n)}\right] \\
+t^{n} \lambda^{-n+(3 k+5) / 2}\left[K_{2} \phi_{1}(\lambda)-\frac{2 K_{3} y_{1}}{(2 k+3)}\right] .
\end{gathered}
$$


Observe that (30b) can (in principle) be inverted to give $\omega(x, y, t)$ if and only if $X_{\omega} \neq 0$; to leading order, this is equivalent to $y_{1} \neq 2 c \alpha^{-1 / 2} /(k+1+2 n)$. Equations $(30 \mathrm{a}, \mathrm{b})$ are valid for all positive times and for all $y_{1}=O(1)$ except in a small neighbourhood of $y_{1}=2 c \alpha^{-1 / 2} /(k+1+2 n)$; note again that $\lambda$ and $k$ must satisfy either of $(16 \mathrm{a}, \mathrm{b})$. The leading term of (30a), namely $t^{2 n-1} F_{1}$, is in fact an asymptotic solution of $\psi_{x x}+\psi_{y y}=0$, and the next term, of $O\left(\lambda^{k+2-2 n} t^{2 n-1}\right)$, can be considered to be a disturbance stream function having the vorticity given by $(30 \mathrm{~b})$. This disturbance is small compared to the leading term for almost all $y_{1}=O(1)$, but is strongly nonlinear in a region described by

$$
\begin{aligned}
y_{1}-\left[4 c \alpha^{-1 / 2}(k+1)\right] /[(k+1+2 n)(k+1-2 n)] & =O\left(\lambda^{k+2}\right) \\
\text { if }(k+2-2 n) & \neq 0 \text { and } \quad(3 k+5-2 n) \neq 0
\end{aligned}
$$

and

$$
\begin{aligned}
y_{1}-\left[4 c \alpha^{-1 / 2}(k+1)\right] /[(k+1+2 n)(k+1-2 n)] & =O\left(\lambda^{k+2} \log \lambda\right) \\
\text { if }(k+2-2 n) & =0 \text { or }(3 k+5-2 n)=0 .
\end{aligned}
$$

We are not interested in the region near $y_{1}=0$ where the disturbance is strongly nonlinear because in this region the leading behaviour of the vorticity function reduces to the known solution (e) of Sec. 1 (the term $y h(\omega, t)$ in (6) becomes negligible in comparison to $g(\omega, t)$ if $\left.y_{1} \rightarrow 0\right)$. Observe from $(30 \mathrm{~b})$ that for $y_{1}=O(1)$ the vorticity is a complex twodimensional time-dependent function that does not correspond to the known solutions (a)-(e) described in Sec. 1. In general, the inversion of (30b) to obtain $\omega(x, y, t)$ must be done numerically.

Case (ib). $k+1-2 n=0$ and (23), (26) hold.

Define $y_{2}=y \lambda^{k+1} t^{-n} / \log \lambda$, and consider the region $y_{2}=O(1)$. Further, let

$$
\begin{array}{r}
F_{2}\left(\lambda, y_{2}\right) \equiv(1 / 2) \lambda^{-k-1}(\log \lambda)^{2}\left[c \alpha^{1 / 2} y_{2}-\alpha y_{2}^{2}\right] \\
+(k+1)^{-1} c \alpha^{1 / 2} y_{2} \lambda^{-k-1} \log \lambda .
\end{array}
$$

We obtain the following expansions for $\Psi$ and $X$, from (29) and (6):

$$
\begin{aligned}
& t^{-k} \Psi(\omega, y, t) \sim F_{2}\left(\lambda, y_{2}\right)-\alpha^{2} y_{2}^{2}(k+2)^{-2} \lambda(\log \lambda)^{2} \\
& +\left[\frac{c \alpha^{3 / 2}(k+3)(3 k+5)}{2(k+1)(k+2)^{3}}\right] y_{2} \lambda \log (\lambda)-(k+1)^{-2} \alpha c^{2} \lambda,
\end{aligned}
$$

and

$$
\begin{aligned}
x=X(\omega, y, t) \sim & (k+1)^{-1} t^{(k+1) / 2} \log (\lambda)\left[c \alpha^{1 / 2}-\alpha y_{2}\right] \\
& -(k+2)^{-2} t^{(k+1) / 2} \lambda^{k+2}\left[\frac{2 \alpha^{2} y_{2} \log (\lambda)}{(2 k+3)}-\frac{c \alpha^{3 / 2}(k+3)}{(k+1)(k+2)}\right] .
\end{aligned}
$$

We may invert (32b) to obtain $\omega(x, y, t)$ in those regions where $X_{\omega} \neq 0$, and this yields $y_{2} \log (\lambda) \neq c \alpha^{-1 / 2} /(k+1)$, to leading order. Note that $\left[t^{k} F_{2}\left(\lambda, y_{2}\right)\right]$ represents an asymptotic solution of $\psi_{x x}+\psi_{y y}=0$, and the remaining terms correspond to a disturbance 
stream function having the vorticity given by $(32 \mathrm{~b})$. This disturbance is strongly nonlinear in the following region near $y_{2}=c \alpha^{-1 / 2}$ :

$$
F_{2}\left(\lambda, y_{2}\right)=O\left(\lambda\{\log \lambda\}^{2}\right)
$$

Observe that we are not interested in the region near $y_{2}=0$ where (III) holds, because in this region the leading behaviour of the vorticity function reduces to the known solution (e) of Sec. 1, as can be seen from (32b). As in case (ia), the vorticity is a complex, two-dimensional time-dependent function in the region of interest, namely $y_{2}=O(1)$.

Case (ii). $k+1+2 n=0$ and (24), (27) hold.

Define $y_{3}=y t^{(k+1) / 2} / \log (\lambda)$ and consider the region $y_{3}=O(1)$. Let

$$
\begin{aligned}
F_{3}\left(\alpha, y_{3}\right) \equiv & -(1 / 2) \lambda^{k+1} \log (\lambda)\left[\left\{\alpha y_{3}^{2}+c \alpha^{1 / 2} y_{3}\right\} \log (\lambda)+(k+1)^{-1}\left\{2 c^{2}-c \alpha^{1 / 2} y_{3}\right\}\right] \\
& +c^{2}(k+1)^{-2} \lambda^{k+1} .
\end{aligned}
$$

The expansion for $\Psi$ reduces to

$$
t^{k+2} \Psi(\omega, y, t) \sim F_{3}\left(\lambda, y_{3}\right)-\lambda^{2 k+3}(\log \lambda)^{2}\left[c_{1} y_{3}^{2}+c_{2} y_{3}+c_{3}\right]
$$

where

$$
\begin{aligned}
& c_{1}=\alpha^{2}(k+2)^{-2} \\
& c_{2}=c \alpha^{3 / 2}(3 k+5)(k+2)^{-2}(2 k+3)^{-1}, \\
& c_{3}=\alpha c^{2}(k+2)^{-1}(2 k+3)^{-1} .
\end{aligned}
$$

The expansion for $X$ is given by

$$
\begin{aligned}
& x=X(\omega, y, t) \\
& \begin{aligned}
& \sim-t^{-(k+1) / 2} \lambda^{k+1} \log (\lambda)\left[\left(\alpha y_{3}+c \alpha^{1 / 2}\right)(k+1)^{-1}-c \alpha^{1 / 2}(k+1)^{-2}(\log \lambda)^{-1}\right. \\
&\left.+2\left\{\alpha^{2} y_{3}(k+2)^{-2}(2 k+3)^{-1}+c \alpha^{3 / 2}(k+2)^{-2}(2 k+3)^{-1}\right\} \lambda^{k+2}\right] .
\end{aligned}
\end{aligned}
$$

Equation $(34 \mathrm{~b})$ can be inverted to give $\omega(x, y, t)$ provided $X_{\omega} \neq 0$, which implies

$$
y_{3} \neq-c \alpha^{-1 / 2}\left[1-(k+1)^{-1}(\log \lambda)^{-1}\right] \text {, to two leading terms. }
$$

Hence, Eqs. (34a, b) are not valid in a small region around the above value of $y_{3}$. It can be verified that $t^{-k-2} F_{3}\left(\lambda, y_{3}\right)$ is an asymptotic solution of $\psi_{x x}+\psi_{y y}=0$, and the remaining terms in $(34 \mathrm{a})$ constitute a strongly nonlinear disturbance in a small region around

$$
y_{3} \sim-c \alpha^{-1 / 2}\left[1-3(k+1)^{-1}(\log \lambda)^{-1}\right] .
$$

This strongly nonlinear region is defined by

$$
F_{3}\left(\lambda, y_{3}\right)=O\left(\lambda^{2 k+3}\{\log \lambda\}^{2}\right)
$$

Essentially the same comments made previously for cases (ia, b) apply. 
Case (iii). $k+3-2 n=0$ and (25), (28) hold.

Define $y_{4}=y \lambda^{k+2} t^{-(k+3) / 2}$, and consider the region $y_{4}=O(1)$. Let

$$
F_{4}\left(\lambda, y_{4}\right) \equiv-(1 / 2) \lambda^{-k-3}\left[\alpha y_{4}^{2}+c \alpha^{1 / 2}(k+1)(k+2)^{-1} y_{4}\right]
$$

The expansion for $\Psi$ reduces to

$$
\begin{gathered}
t^{-k-2} \Psi(\omega, y, t) \sim F_{4}\left(\lambda, y_{4}\right)+\lambda^{-1} \log (\lambda)\left[\alpha c^{2}(k+2)^{-2}+c_{4} y_{4}\right] \\
+\lambda^{-1}\left[c_{5}+c_{6} y_{4}-\alpha^{2}(k+2)^{-2} y_{4}^{2}\right],
\end{gathered}
$$

where the constants $c_{4}, c_{5}$, and $c_{6}$ are defined by

$$
\begin{aligned}
& c_{4}=(1 / 2) \alpha^{3 / 2} c(3 k+5)(k+1)^{-1}(k+2)^{-2}, \\
& c_{5}=\alpha c^{2}(k+4)(k+2)^{-3}, \\
& c_{6}=(1 / 2) \alpha^{3 / 2} c(k+1)^{-2}(k+2)^{-3}\left[k(k+3)+4(k+1)^{2}\right] .
\end{aligned}
$$

In $(35 a)$ the vorticity $\omega(x, y, t)$ is defined implicitly by

$$
\begin{aligned}
x=X(\omega, y, t) \sim-t^{(k+3) / 2}\left[\lambda^{-1}\{\right. & \left.\alpha(k+1)^{-1} y_{4}+c \alpha^{1 / 2}(k+2)^{-1}\right\} \\
& \left.+c_{7} \lambda^{k+1} \log (\lambda)+\lambda^{k+1}\left\{c_{8}+c_{9} y_{4}\right\}\right],
\end{aligned}
$$

where the constants $c_{7}, c_{8}$, and $c_{9}$ are defined by

$$
\begin{aligned}
& c_{7}=-\alpha^{3 / 2} c(k+1)^{-1}(k+2)^{-2}, \\
& c_{8}=-\alpha^{3 / 2} c k(k+1)^{-2}(k+2)^{-3}, \\
& c_{9}=2 \alpha^{2}(k+2)^{-2}(2 k+3)^{-1} .
\end{aligned}
$$

The requirement $X_{\omega} \neq 0$ implies that Eqs. (35a, b) are not valid in a small region around $y_{4}=c \alpha^{-1 / 2}(k+2)^{-1}$. The term $t^{k+2} F_{4}\left(\lambda, y_{4}\right)$ of $(35 \mathrm{a})$ is an asymptotic solution of $\psi_{x x}+\psi_{y y}=0$, and the disturbance stream function displayed is strongly nonlinear in a region defined by

$$
y_{4}+c \alpha^{-1 / 2}(k+1)(k+2)^{-1}=O\left(\lambda^{k+2} \log \lambda\right) .
$$

Again, similar comments to those made for cases (ia, b) apply.

3. Concluding remarks. In cases (ia, b), (ii), and (iii), we have obtained unsteady asymptotic solutions of the Euler equations that are formally valid for all $t>0$ in the region $h(\omega, t)=H(\omega t) \rightarrow 0^{+}$and for almost all $y_{i}=O(1)$ (where $i=1, \ldots, 4$ ); a small region around a critical value of $y_{i}$ where $X_{\omega}=0$ must be excluded to avoid a singularity in the transformation (2). Note that $\omega_{x}$ becomes unbounded as $y_{i}$ approaches this critical value. The interesting features of these solutions can be summarized as follows.

(1) We have obtained a general class of unsteady asymptotic solutions of the twodimensional Euler equations. To see this, note that the solution in case (ia) contains two free parameters $k$ and $n$, while cases (ib), (ii), and (iii) contain one free parameter $k$; 
further, the results are valid subject to either of Eqs. (16a, b). Each different permitted combination of $k$ and $n$ yields a qualitatively different vorticity function $\omega(x, y, t)$.

(2) The vorticity function $\omega$, here defined by the implicit relationship $x=X(\omega, y, t)$, has a complicated dependence on $(x, y, t)$ in contrast to previously known exact solutions. The generality and complexity of the results obtained make them theoretically interesting and possibly useful in applications. To the author's knowledge, this level of generality and complexity has not been attained in previous descriptions of analytical solutions of the Euler equations.

(3) As noted earlier, our results may be interpreted as constituting disturbance stream functions for "basic" flows that satisfy $\psi_{x x}+\psi_{y y}=0$. These disturbances are strongly nonlinear in the regions $(\mathrm{I}-\mathrm{V})$ defined in Sec. 2, and weakly nonlinear elsewhere in the region of validity. Even the weakly nonlinear region is interesting because the basic flow here is strongly two-dimensional and time-dependent.

(4) Subject to further restrictions, the results obtained here are in fact valid asymptotic solutions of the full Navier-Stokes equations (which have the additional term $\nu\left(\omega_{x x}+\omega_{y y}\right)$ on the right-hand side of (1a), where $\nu$ is the kinematic viscosity). The extra restriction needed is that the leading part of the viscous terms be negligible in comparison to all the terms on the left-hand side of (1a) that have been taken into account. For example, it can be verified that for case (ia), the leading two terms of the expansion for $\omega_{t}$ on the left-hand side of (1a) dominate the viscous terms provided

$$
\lambda^{2 k+3-2 n} t^{2 n-1} \gg \nu \text { and } y_{1}=O(1) .
$$

If the above restrictions hold, the results for case (ia) are valid asymptotic solutions of the full Navier-Stokes equations.

(5) We may require that Eqs. (6-10) hold to still higher orders than considered here; we have not done so only because of the algebraic complexities involved.

We close with a couple of remarks on the possibilities for further research. Firstly, note that we have not by any means provided the complete solution of the system (7-10). Even within the limitations of the transformation (11), Eq. (10) reduces to the formidable nonlinear ordinary differential equation (12), whose general solution appears elusive. Secondly, we may seek alternatives to Eq. (6) for which interesting asymptotic or exact solutions may be deduced by the methods described here.

Appendix A. Previously known solutions. Here we briefly consider the known solutions (a)-(e) mentioned in Sec. 1.

(a) Let $\omega=f(r, t)$, where $(r, \theta)$ are polar coordinates with $r=\left(x^{2}+y^{2}\right)^{1 / 2}$ and $\theta=\tan ^{-1}(y / x)$. Let $\psi=a(r, t) \theta+b(r, t)$. Equation (1a) reduces to

$$
f_{t}+a r^{-1} f_{r}=0 \text {. }
$$

From (1b), we find

$$
\theta\left\{a_{r r}+r^{-1} a_{r}\right\}+b_{r r}+r^{-1} b_{r}=-f .
$$

The above equation will hold if $a(r, t)$ and $b(r, t)$ satisfy

$$
a_{r r}+r^{-1} a_{r}=0 \quad \text { and } \quad b_{r r}+r^{-1} b_{r}=-f .
$$


Equations (A.1) and (A.2) determine the functions $f, a$, and $b$.

(b) Again consider the polar coordinates $(r, \theta)$. We take $\omega=f(\theta, t)$ and $\psi=r^{2} g(\theta, t)$. Equations $(1 \mathrm{a}, \mathrm{b})$ then reduce to the following:

$$
f_{t}-2 g f_{\theta}=0 \text { and } g_{\theta \theta}+4 g=-f \text {. }
$$

Equation (A.3) determines $f$ and $g$; special explicit solutions to (A.3) can be found.

(c) Take $\omega=f(\xi)$, where $\xi=x+g(t) y$. Further, assume $\psi$ in the form $\psi=a(t) y^{2}+$ $h(\xi, t)$. Then Eqs. (1a, b) reduce to

$$
g^{\prime}(t)=-2 a(t) \text { and } h_{\xi \xi}=-\left(1+g^{2}\right)^{-1}(f+2 a) .
$$

Note that $g(t)$ and $f(\xi)$ can be specified arbitrarily.

(d) Take $\omega=f(x, y) / t$ and $\psi=g(x, y) / t$. Then Eqs. (1a, b) reduce to

$$
g_{y} f_{x}-g_{x} f_{y}=f \quad \text { and } \quad g_{x, x}+g_{y, y}=-f .
$$

Special explicit solutions to the above system can be found.

(e) Let $\omega=f(x, t)$. Then (1a) implies $\psi_{y}=-f_{t} / f_{x}$, from which $\psi=a(x, t)-\left\{y f_{t} / f_{x}\right\}$. Upon substitution into (1b), we obtain $-y\left(\partial^{2} / \partial x^{2}\right)\left\{f_{t} / f_{x}\right\}+a_{x x}=-f$, from which

$$
\left(\partial^{2} / \partial x^{2}\right)\left\{f_{t} / f_{x}\right\}=0 \text { and } a_{x, x}=-f .
$$

The solution of (A.6) for $f$ and $a$ is straightforward.

Appendix B. Derivation of Eq. (17) of Sec. 2. Assume that either of (16a, b) holds. Let us try the following expansion for $E^{\prime}(\lambda)$ to leading order:

$$
E^{\prime}(\lambda) \sim E_{0}^{\prime}(\lambda)=\beta \lambda^{3 k+4},
$$

where $\beta$ is a constant to be determined. From (13) we then obtain

$$
H^{\prime}(\lambda) \sim H_{0}^{\prime}(\lambda)+H_{1}^{\prime}(\lambda)+E_{0}^{\prime}(\lambda) .
$$

where $H_{0}^{\prime}(\lambda)$ and $H_{1}^{\prime}(\lambda)$ are obtained from $(15 \mathrm{a}, \mathrm{b})$ and $E_{0}^{\prime}$ is given by (B.1). Upon substitution of (B.2) in (12), we find that the leading two terms corresponding to (14a, b) cancel out as expected. Further, terms of $O\left(\lambda^{4 k+1}\right)$ in (12) also cancel themselves out; it can be verified that these terms are

$$
H_{0}^{2}\left\{\lambda H_{0}^{\prime} H_{0}^{\prime \prime \prime}-\lambda\left(H_{0}^{\prime \prime}\right)^{2}+H_{0}^{\prime} H_{0}^{\prime \prime}\right\}+2 \lambda\left(H_{0}^{\prime}\right)^{-1}-2 H_{0}\left(H_{0}^{\prime}\right)^{2}\left\{H_{0}^{\prime}+\lambda H_{0}^{\prime \prime}\right\}=0 .
$$

Therefore, $E_{0}^{\prime}(\lambda)$ must be chosen so as to cancel out terms of $O\left(\lambda^{4 k+3}\right)$ in (12), and we find that $E_{0}^{\prime}$ satisfies the following equation:

$$
\begin{aligned}
& \lambda\left[H_{0}^{\prime} E_{0}^{\prime \prime \prime}+H_{0}^{\prime \prime \prime} E_{0}^{\prime}-2 H_{0}^{\prime \prime} E_{0}^{\prime \prime}\right]+H_{0}^{\prime} E_{0}^{\prime \prime}+H_{0}^{\prime \prime} E_{0}^{\prime} \\
& =6 \lambda\left(H_{0}^{\prime}\right)^{2} H_{1}^{\prime}-H_{1}^{\prime} H_{1}^{\prime \prime}-\lambda\left[H_{1}^{\prime} H_{1}^{\prime \prime \prime}-\left(H_{1}^{\prime \prime}\right)^{2}\right] .
\end{aligned}
$$

Upon substituting (B.1) and (15a, b) into (B.3) it is a straightforward matter to verify that the constant $\beta$ in (B.1) must be given by

$$
\beta=3 \alpha^{3}(k+2)^{-4} .
$$

Hence, Eq. (17) follows. The crucial point is that terms of $O\left(\lambda^{4 k+1}\right)$ in (12), which dominate the terms in (B.3) if (16a) holds, cancel themselves out as shown above. 


\section{REFERENCES}

[1] G. K. Batchelor, An Introduction to Fluid Dynamics, Cambridge University Press, 1967, Chapter 7

[2] R. Berker, Intégration des équations du mouvement d'un fluide visqueux incompressible, in Handbuch der Physik, ed. S. Flügge, vol. VIII/2, Springer-Verlag, Berlin, 1963, pp. 1-384

[3] C. Y. Wang, Exact solutions of the unsteady Navier-Stokes equations, Appl. Mech. Rev. 42, S269$282(1989)$

[4] K. B. Ranger, A complex variable integration technique for the two-dimensional Navier-Stokes equations, Quart. Appl. Math. 49, 555-562 (1991)

[5] K. B. Ranger, Solutions of the Navier-Stokes equations in implicit form, Quart. Appl. Math. 50, 793-800 (1992) 